\title{
Optical diagnostics of bile duct tissues state with tumor compression
}

Kandurova, Ksenia, Dremin, Viktor, Zherebtsov, Evgeny, Potapova, Elena, Filina, Mariya, et al.

Ksenia Y. Kandurova, Viktor V. Dremin, Evgeny A. Zherebtsov, Elena V. Potapova, Mariya A. Filina, Andrey V. Dunaev, Andrian V. Mamoshin, Alexandr L. Alyanov, Vadim F. Muradyan, "Optical diagnostics of bile duct tissues state with tumor compression," Proc. SPIE 11065, Saratov Fall Meeting 2018: Optical and Nano-Technologies for Biology and Medicine, 1106508 (3 June 2019); doi: 10.1117/12.2522670 


\title{
Optical diagnostics of bile duct tissues state with tumor compression
}

\author{
Ksenia Y. Kandurova ${ }^{a^{*}}$, Viktor V. Dremin ${ }^{a}$, Evgeny A. Zherebtsov ${ }^{\mathrm{a}, \mathrm{b}}$, Elena V. Potapova ${ }^{\mathrm{a}}$, \\ Mariya A. Filina ${ }^{\mathrm{a}}$, Andrey V. Dunaev ${ }^{\mathrm{a}}$, Andrian V. Mamoshin ${ }^{\mathrm{a}, \mathrm{c}}$, Alexandr L. Alyanov ${ }^{\mathrm{a}, \mathrm{c}}$, \\ Vadim F. Muradyan ${ }^{c}$ \\ ${ }^{a}$ Research and Development Center of Biomedical Photonics, Orel State University named after I.S. \\ Turgenev, Orel, Russia; \\ ${ }^{\mathrm{b}}$ OPEM, University of Oulu, Oulu, Finland; \\ ${ }^{c}$ Orel Regional Clinical Hospital, Orel, Russia
}

\begin{abstract}
The paper presents the results of in vivo experimental measurements of the endogenous fluorescence and blood perfusion in common bile duct tissues in obstructive jaundice patients during minimally invasive surgical operations. Nowadays the type of interventions become more widespread for treating hepatopancreatobiliary pathologies. An effective feedback which can be established by one or more optical diagnostic channels integrated into the standard instruments for minimally invasive manipulations would be of particular value to practical surgeons. To collect reference data, an experimental study has been conducted using a fiber optical probe with fluorescence spectroscopy and laser Doppler flowmetry channels. For every measurement, the areas of interest were different areas of common bile duct obstructed by a malignant tumor. Obtained results demonstrated significant variety registered intensities of fluorescence spectra and values of blood perfusion. Tested fiber optical endoscopic system showed sensitivity to the tissue state in the investigated area, so the two modal approach seems to be promising for further research and implementation in clinical practice.
\end{abstract}

Keywords: optical diagnostics, optical biopsy, spectroscopy, fluorescence spectroscopy, laser Doppler flowmetry, minimally invasive surgery, common bile duct, obstructive jaundice, tumor compression

\section{INTRODUCTION}

Currently, visualization and analysis of malignant, inflamed and necrotic tissues remain one of the urgent problems of biophotonics applications for surgery. In particular, this of great importance in diagnosing the causes of obstructive jaundice. This syndrome is caused by obstruction of common bile duct preventing normal drainage of bile from the liver to the small intestines.

Obstructive jaundice is one of the typical symptoms manifested in patients with malignant and benign tumors of hepatopancreatobiliary organs, cholelithiasis, cicatrical changes, pancreatitis, etc. ${ }^{1}$ Speaking of tumors, the primary cause is pancreatic cancer ${ }^{2}$. The less occurring origin of obstructive jaundice is gallbladder cancer, cholangiocarcinoma and hepatocellular carcinoma ${ }^{3,4}$.

The difficulties of early diagnosis cause increasing complications probability and mortality rates. Hepatopancreatobiliary organs cancer shows no clinical signs at early stages ${ }^{5,6}$. Patients are admitted to hospitals due to complications. An abundant one of them is the obstructive jaundice which is usually diagnosed at the late stages of the tumor development. Early diagnosis of cancer is a difficult task in that area. In most cases, the collection of factors affect the duration and effectiveness of the treatment measures.

In this regard, the problem of increasing the quality of diagnosis during the treatment becomes more relevant, especially for minimally invasive surgery. Such techniques become more widespread for diagnosing and surgical treatment of the pathologies of abdominal organs. Compared to conventional open surgery, minimally invasive surgery allows one to decrease the operative morbidity and mortality, as well as to lower the rehabilitation cost and recovery time ${ }^{7}$. However, the challenges of the intraoperative collection and processing of the diagnostic information for these techniques is not solved well. The process of identification of new diagnostic criteria for increasing the accuracy of diagnosis of malignant tissues in minimally invasive surgery remains relevant.

*kandkseniya@gmail.com; phone +7 910 2682946; http://www.bmecenter.ru/en 
At preresent, the reference method using as a gold standard for tissue type recognition is a histological examination of samples obtained by biopsy. However, obtaining results takes some time. Furthermore, the method itself is an origin for several types of complications. Existing widespread diagnostic methods allows either obtain the information about the state of pathological tissue later or obtain intraoperatively the information about anatomical and morphological features rather than tissue state. The method combining the advantages of both approaches is necessary for further development of minimally invasive technologies. Implementation of new methods in clinical practice will improve the quality of diagnostics and treatment.

One of the rapidly developing areas of research is optical non-invasive methods, which are widely used in various areas, including medicine. In particular, one of the dynamically developing direction is applying these methods to determine the state of biological tissues during surgical operations ${ }^{8}$. The various spectroscopic and imaging techniques involved in this area of research are called "optical biopsy". The main advantage of these methods is a possibility to study biological tissues in vivo in real time. These methods allow for evaluating metabolic processes in tissues, chromophores content, blood perfusion and oxygenation. The use of optical methods in minimally invasive abdominal surgery can increase the prevalence and effectiveness of these interventions in clinical practice by providing additional diagnostic information for surgeon intraoperatively.

One of the most popular optical spectroscopic methods for in vivo diagnostics is fluorescence spectroscopy (FS). The method is based on probing a living biological tissue with UV or visible optical radiation to record the spectrum of its fluorescence emission. In microscopy, FS is actively used for studying the structure and biochemical properties of cells and tissues. The method can be used to analyze the emission of both fluorescence contrast substances and endogenous fluorophores $\left(\mathrm{NAD}(\mathrm{P}) \mathrm{H}\right.$, flavins, collagen, elastin, porphyrins, lipofuscin, etc. $\left.{ }^{9}\right)$ The amount of these substances, especially NADH and FAD coenzymes shows the rate of metabolic activity of tissues ${ }^{10}$. The reduced form of NADH has a fluorescence intensity maximum at a wavelength of $490 \mathrm{~nm}$ when excited with $365 \mathrm{~nm}$ radiation. Oxidized FAD has a maximum of fluorescence intensity in the range of 520-540 nm when excited with $450 \mathrm{~nm}$ radiation ${ }^{11}$. The accumulation of these coenzymes in mitochondria during the tricarbonic acid cycle is related to the processes of oxidative phosphorylation. Therefore, the intensity of metabolic processes can be estimated according to the content of NADH and FAD which affects fluorescence spectra. As the optical properties of tissues are sensitive to the metabolism conditions, FS has opportunities to determine destructive inflammatory, malignant and necrotic processes in biological tissues.

More accurate and valuable information for diagnosis and treatment can be acquired with the multimodal approach combining several optical techniques to gain their advantages ${ }^{12,13}$. However, the application of even one method in modern minimally invasive surgery is associated with some technological and methodological challenges.

Thus, this work aimed to study the autofluorescence of malignant and non-malignant tissues of the common bile duct in obstructive jaundice patients during minimally invasive surgical operations to obtain the information about the metabolic activity of cells and state of tissues.

\section{MATERIALS AND METHODS}

The scheme of experimental setup is shown in Figure 1. The most parts of the setup were developed jointly with SPE "LAZMA" Ltd (Moscow, Russia). The setup implements two optical methods. The first one is FS at excitation wavelengths of $365 \mathrm{~nm}$ and $450 \mathrm{~nm}$ for assessing metabolic activity by registering the fluorescence of NADH and FAD coenzymes. The spectra were registered with a spectrometer in the range of 350-820 nm. The second method is laser Doppler flowmetry. This method is based on probing the biological tissue with laser radiation and analyzing the Doppler shift caused by light scattered and reflected by erythrocytes moving in microcirculatory bed. LDF was used for assessing blood flow parameters ${ }^{14}$ of tissues in addition to FS, as it is widely known that blood affects recorded spectra ${ }^{15}$.

The experimental study involved 20 patients of Orel Regional Clinical Hospital department of X-ray-surgical methods of diagnosis and treatment aged $67 \pm 2$ years diagnosed obstructive jaundice caused by malignant tumors of hepatopancreatobiliary organs. The studies were approved by Ethics committee Orel State University (record of the meeting №10 of 16.11.2017). All patients signed an informed consent indicating their voluntary willingness to participate in the study. The measurements were performed during prescribed diagnostic and therapeutic minimally invasive surgical operations under ultrasonic and fluoroscopic control. 
Pairs of fluorescence spectra were recorded in two areas of interest, which are shown in Figure 1. The aim was to compare the data obtained at the area, which was blocked by the malignant tumor, and another area located higher than the first one. LDF signals were recorded simultaneously with fluorescence spectra. Each wavelength spectra were registered three times in each area and averaged for further processing.

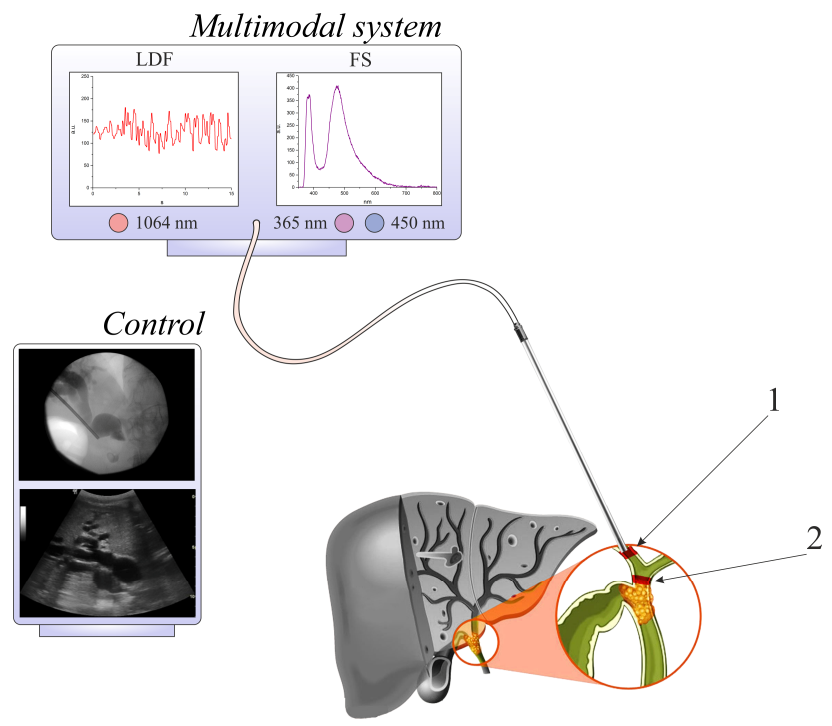

Figure 1. The schematic illustration of the experimental setup. The areas of interest are: 1 - area non-blocked by tumor; $2-$ area blocked by a tumor

In order to correctly compare the data obtained from different areas, obtained fluorescence intensities were normalized by backscattered radiation using the following formula:

$$
I_{\text {norm }}(\lambda)=\frac{I(\lambda)}{I_{\max \text { source }}}
$$

$I(\lambda)$ is fluorescence intensity at $\lambda \mathrm{nm}$ wavelength, $I_{\max \text { source }}$ is the maximum intensity of backscattered radiation. This formula allows compensating significant scatter between raw fluorescence intensities in observed spectra.

\section{RESULTS AND DISCUSSION}

The averaged fluorescence spectra are shown in Figure 2.

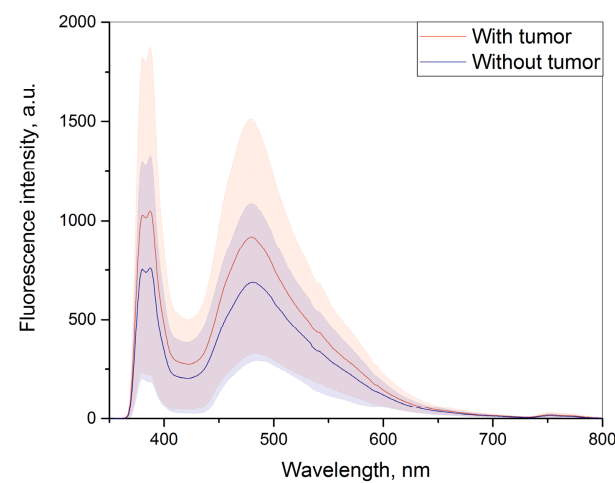

(a)

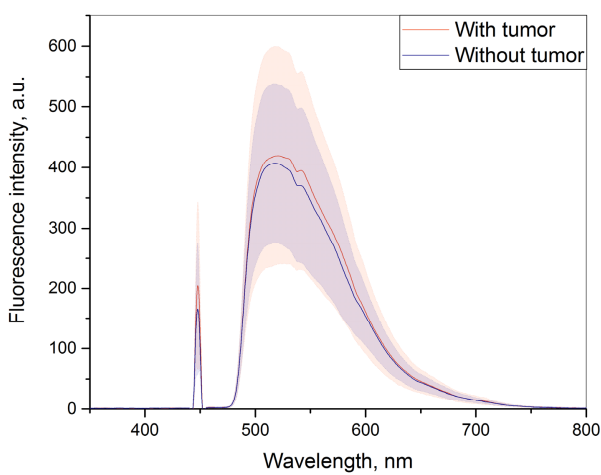

(b)

Figure 2. The averaged fluorescence spectra at excitation wavelength $365 \mathrm{~nm}$ (a) and $450 \mathrm{~nm}$ (b) 
The high variability was observed in the obtained data, which can be seen by the colored areas of the signal variation. Despite having several cases of 1.5-3 times difference in intensities between the areas, mean fluorescence spectra showed no significant difference. However, fluorescence intensities were higher in tumor areas, which is seen more clearly at $365 \mathrm{~nm}$ spectra (Fig. 2, a).

In order to correctly compare the obtained data from different areas and under different excitation wavelengths, the spectra were normalized by backscattered radiation. The results of normalizing and averaging the data on both wavelengths are shown in Figure 3. The high variability still can be seen as well as no significant difference between the mean fluorescence spectra. However, the fluorescence intensity maxima became higher in the non-blocked area. It total, the fluorescence under $450 \mathrm{~nm}$ excitation is more intense.

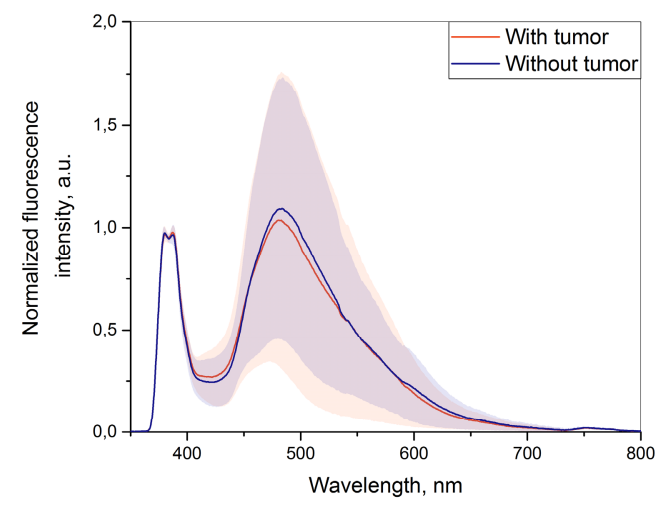

(a)

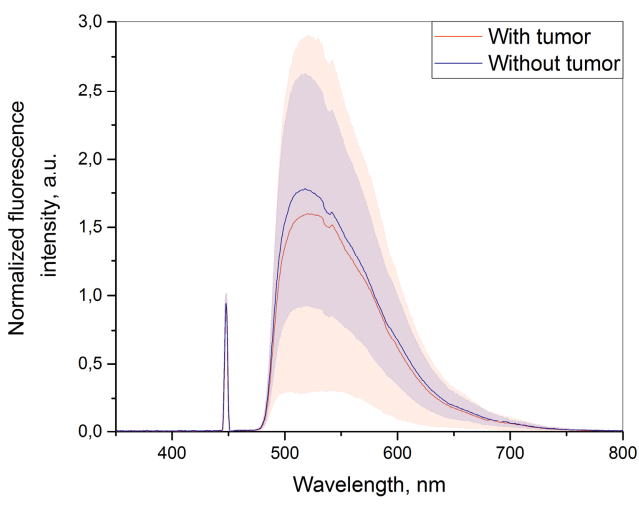

(b)

Figure 3. The averaged fluorescence spectra after normalizing at excitation wavelength $365 \mathrm{~nm}$ (a) and $450 \mathrm{~nm}$ (b)

After more detailed processing of the fluorescence spectra, two main types of spectrum were identified among $365 \mathrm{~nm}$-excited fluorescence spectra. The spectra obtained under the excitation wavelength of $450 \mathrm{~nm}$ showed no such result. The half of them fell into the first type (Fig. 4, a), the rest of the spectra were considered the second type (Fig. 4, b). It was supposed that averaging all the spectra levels the difference. Therefore, these cases should be considered separately. The first type spectra demonstrated higher fluorescence intensity in non-compressed areas. By comparison, the results of the second group showed more intense fluorescence in the blocked area.

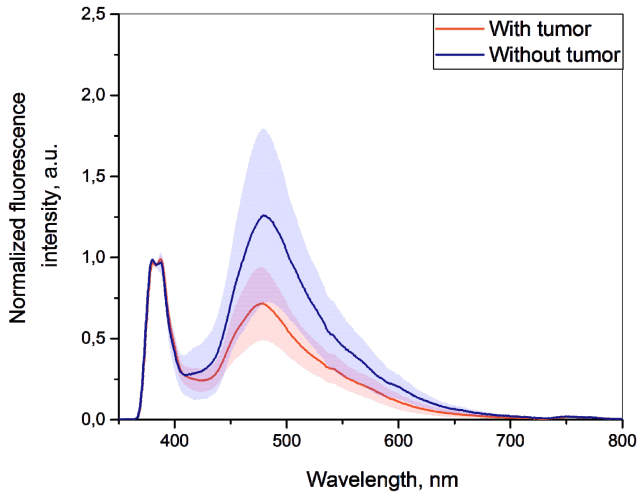

(a)

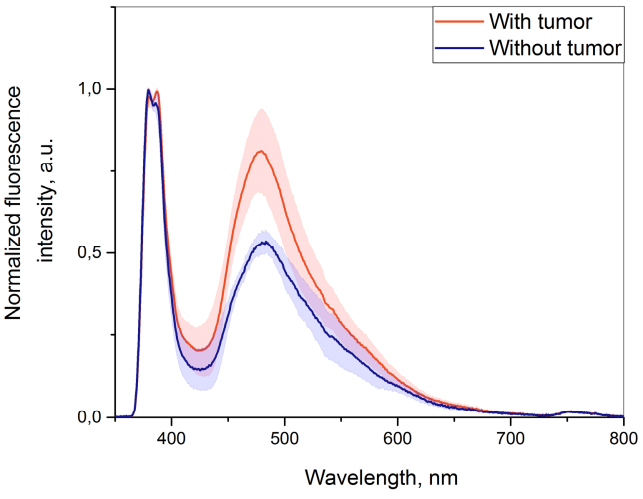

(b)

Figure 4. The normalized averaged spectra at excitation wavelength $365 \mathrm{~nm}$ in first (a) and second type (b)

During the in vivo studies, it was not impossible to certainly determine whether the exophytic and intracranial growth of tumor had occurred or not. Analysis of literature showed that usually registered fluorescence intensity under ultraviolet excitation has higher values in the intact area rather than in tumor ${ }^{16-20}$. Thus, it is possible to put forward a hypothesis that the first type spectra demonstrate the growth of tumor into the walls of the common bile duct. At another point, the increasing of fluorescence intensity in the blocked area in the second group might point out at the decreased blood supply caused by tumor obstruction outside the common bile duct. Also, it is possible that the increased presence of 
collagen in the intercellular matrix of the muscle layer of common bile duct wall, the presence of blood and bile in the studied area could affect the recorded fluorescence spectra.

The average 1-minute long LDF signals recorded in two areas during interventions are shown in Figure 5.

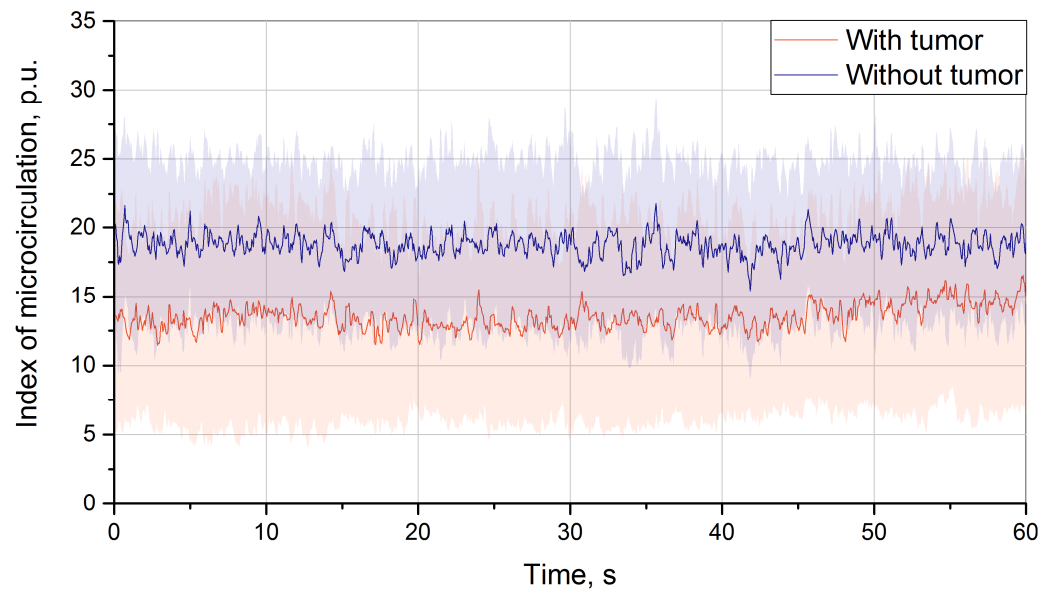

Figure 5. The averaged LDF signals of the areas of interest

Obtained data has showed a decrease in blood microcirculation in the tumor lesions area compared to the area not affected by the cancer process. This can show reduced blood supply of the blocked area caused by tumor obstruction as well. Some methodological difficulties, such as pressure caused by the probe ${ }^{21}$, anatomical features of the studied area, movements and inconvenience for the patient during the procedure, caused the short duration of LDF signals recordings.

\section{CONCLUSION}

Generally, the obtained data showed the possibility and greater prospect for combined applying of the FS and LDF methods in minimally invasive surgery of the common bile duct. The results showed high individual variability as well as sensitivity to the area of the research. It is supposed that the results were significantly affected by some factors including the presence of blood and other substances, the phase of the pathological process, anatomical features, and shortcomings of existing control methods for minimally invasive surgery. Further research is required to address these issues. The use of an additionalmeasuring channel such as diffuse reflectance spectroscopy channel is planned to be used to simultaneously evaluate the influence of the variable blood content on the registered fluorescence intensity ${ }^{22}$.

The multimodal approach combining two optical diagnostic methods seems promising for further research. To improve the quality of diagnostic information and optimize the diagnostic and treatment algorithm, it is proposed to extend some methods by adding diffuse reflection spectroscopy method and imaging techniques. Moreover, at the initial stages of the research, a combination of optical biopsy and traditional biopsy followed by a histological and cytological examination of biological tissue is planned. This will make it possible to use traditional biopsy not only as a method of verification of pathological changes in tissues but also as a reference for more accurate interpretation of the results obtained by optical diagnostic methods. This will form the basis for the development of diagnostic criteria for application in clinical practice.

\section{ACKNOWLEDGEMENTS}

This study was supported by the Russian Science Foundation under project № 18-15-00201.

\section{REFERENCES}

[1] Stinton, L. M. and Shaffer, E. A., "Epidemiology of gallbladder disease: Cholelithiasis and cancer," Gut Liver 6(2), 172-187 (2012). 
[2] Vincent, A., Herman, J., Schulick, R., Hruban, R. H. and Goggins, M., "Pancreatic cancer," Lancet 378(9791), 607-620 (2011).

[3] Qin, L.-X. and Tang, Z.-Y., "Hepatocellular carcinoma with obstructive jaundice: Diagnosis, treatment and prognosis," World J. Gastroenterol. 9(3), 385-391 (2003).

[4] Hundal, R. and Shaffer, E. A., "Gallbladder cancer: Epidemiology and outcome," Clin. Epidemiol. 6(1), 99-109 (2014).

[5] Boj, S. F., Hwang, C.-I., Baker, L. A., Chio, I. I. C., Engle, D. D., Corbo, V., Jager, M., Ponz-Sarvise, M., Tiriac, H., Spector, M. S., Gracanin, A., Oni, T., Yu, K. H., Van Boxtel, R., Huch, M., Rivera, K. D., Wilson, J. P., Feigin, M. E., Öhlund, D., et al., "Organoid models of human and mouse ductal pancreatic cancer," Cell 160(12), 324-338 (2015).

[6] Shen, Z., Tian, L. and Wang, X., "Treatment of pancreatic head cancer with obstructive jaundice by endoscopy ultrasonography-guided gastrojejunostomy: A case report and literature review," Medicine (Baltimore). 97(28), e11476 (2018).

[7] Chahal, P. and Baron, T. H., "Endoscopic palliation of cholangiocarcinoma," Curr. Opin. Gastroenterol. 22(5), 551-560 (2006).

[8] Kennedy, G. T., Okusanya, O. T., Keating, J. J., Heitjan, D. F., Deshpande, C., Litzky, L. A., Albelda, S. M., Drebin, J. A., Nie, S., Low, P. S. and Singhal, S., "The optical biopsy: A novel technique for rapid intraoperative diagnosis of primary pulmonary adenocarcinomas," Ann. Surg. 262(4), 602-609 (2015).

[9] Croce, A. C. and Bottiroli, G., "Autofluorescence spectroscopy and imaging: A tool for biomedical research and diagnosis," Eur. J. Histochem. 58(4), 320-337 (2014).

[10] Tuchin, V. V., "Handbook of Optical Biomedical Diagnostics,” Handb. Biomed. Diagnostics (2002).

[11] Bartolomé, F. and Abramov, A. Y., "Measurement of mitochondrial nadh and fad auto fluorescence in live cells," Methods Mol. Biol. 1264, 263-270 (2015).

[12] Dremin, V. V, Zherebtsov, E. A., Sidorov, V. V, Krupatkin, A. I., Makovik, I. N., Zherebtsova, A. I., Zharkikh, E. V, Potapova, E. V, Dunaev, A. V, Doronin, A. A., Bykov, A. V, Rafailov, I. E., Litvinova, K. S., Sokolovski, S. G. and Rafailov, E. U., "Multimodal optical measurement for study of lower limb tissue viability in patients with diabetes mellitus," J. Biomed. Opt. 22(8) (2017).

[13] Dremin, V. V, Sidorov, V. V, Krupatkin, A. I., Galstyan, G. R., Irina, N., Zherebtsova, A. I., Zherebtsov, E. A., Dunaev, A. V and Zera, N., "The blood perfusion and NADH / FAD content combined analysis in patients with diabetes foot," SPIE Proc. (2016).

[14] Dunaev, A. V., Sidorov, V. V., Krupatkin, A. I., Rafailov, I. E., Palmer, S. G., Stewart, N. A., Sokolovski, S. G. and Rafailov, E. U., "Investigating tissue respiration and skin microhaemocirculation under adaptive changes and the synchronization of blood flow and oxygen saturation rhythms," Physiol. Meas. (2014).

[15] Dremin, V. V, Zherebtsov, E. A., Rafailov, I. E., Vinokurov, A. Y., Novikova, I. N., Zherebtsova, A. I., Litvinova, K. S. and Dunaev, A. V., "The development of attenuation compensation models of fluorescence spectroscopy signals,” Prog. Biomed. Opt. Imaging - Proc. SPIE 9917 (2016).

[16] Anidjar, M., Ettori, D., Cussenot, O., Meria, P., Desgrandchamps, F., Cortesse, A., Teillac, P., Le Duc, A. and Avrillier, S., "Laser induced autofluorescence diagnosis of bladder tumors: Dependence on the excitation wavelength," J. Urol. (1996).

[17] Bogomolov, A., Belikova, V., Zabarylo, U. J., Bibikova, O., Usenov, I., Sakharova, T., Krause, H., Minet, O., Feliksberger, E. and Artyushenko, V., "Synergy effect of combining fluorescence and mid infrared fiber spectroscopy for kidney tumor diagnostics," Sensors (Switzerland) (2017).

[18] Koenig, F., Mcgovern, F. J., Althausen, A. F., Deutsch, T. F. and Schomacker, K. T., "Laser induced autofluorescence diagnosis of bladder cancer," J. Urol. (1996).

[19] Mayinger, B., Jordan, M., Horner, P., Gerlach, C., Muehldorfer, S., Bittorf, B. R., Matzel, K. E., Hohenberger, W., Hahn, E. G. and Guenther, K., "Endoscopic light-induced autofluorescence spectroscopy for the diagnosis of colorectal cancer and adenoma," J. Photochem. Photobiol. B Biol. (2003).

[20] Palmer, S., Litvinova, K., Dunaev, A., Yubo, J., McGloin, D. and Nabi, G., "Optical redox ratio and endogenous porphyrins in the detection of urinary bladder cancer: A patient biopsy analysis," J. Biophotonics 10(8), 10621073 (2017).

[21] Zherebtsov, E. A., Kandurova, K. Y., Seryogina, E. S., Kozlov, I. O., Dremin, V. V, Zherebtsova, A. I., Dunaev, A. V and Meglinski, I., "The influence of local pressure on evaluation parameters of skin blood perfusion and fluorescence,” Prog. Biomed. Opt. Imaging - Proc. SPIE 10336 (2017). 
[22] Zherebtsov, E., Dremin, V., Zharkikh, E., Zherebtsova, A., Rafailov, I., Dunaev, A., Bazieva, N. and Rafailov, E., "Fibre optic probe for fluorescence diagnostics with blood influence compensation," SPIE BiOS 10493, 6, SPIE (2018). 